\title{
RR Lyrae Variables in the Globular Cluster M55. The First Evidence for Nonradial Pulsations in RR Lyr Stars
}

\author{
A. Olech ${ }^{1}$, J. Kaluzny ${ }^{1}$, I. B. Thompson ${ }^{2}$, W. Pych $^{1}$, W. Krzemiński ${ }^{2}$, \\ A. Schwarzenberg-Czerny ${ }^{3}$
}

\begin{abstract}
We present the results of a photometric study of RR Lyrae variables in the field of the globular cluster M55. We have discovered nine new RR Lyrae stars, increasing the number of known variables in this cluster to 15 objects. Five of the newly discovered variables belong to Bailey type RRc and two to type RRab. Two background RRab stars are probable members of the Sagittarius dwarf galaxy. Fourier decomposition of the light curves was used to derive basic properties of the present sample of RR Lyrae variables. From an analysis of the RRc variables we obtain a mean mass of $M=0.53 \pm 0.03 M_{\odot}$, luminosity $\log L=1.75 \pm 0.01 L_{\odot}, T_{\text {eff }}=7193 \pm 27 \mathrm{~K}$, and helium abundance $Y=0.27 \pm 0.01$. We also derive the value of the color excess for M55 to be equal to $E(B-V)=0.11 \pm 0.03$. We estimate the values of the visual apparent distance moduli to be $13.65 \pm 0.11$ from RRab stars and $13.91 \pm 0.08$ from RRc stars. The light curves of three of the $R R c$ variables exhibit changes in amplitude of over $0.1 \mathrm{mag}$ on time scales of less than a week, rather short for the Blazhko effect, but with no evidence for another radial pulsational frequency. However, we do detect other periodicities which are clearly visible in the light curve after removing variations with the first overtone radial frequency. This is strong evidence for the presence of nonradial pulsations, a behavior common for $\delta$ Scuti stars, but not yet observed among RR Lyr variables.
\end{abstract}

\footnotetext{
${ }^{1}$ Warsaw University Observatory, AI. Ujazdowskie 4, 00-478 Warszawa, Poland

${ }^{2}$ Carnegie Institution of Washington, 813 Santa Barbara Street, Pasadena, CA 91101, USA

${ }^{3}$ Astronomical Observatory of Adam Mickiewicz University, ul. Słoneczna 36, Poznań, Poland
} 